\section{Case Reports in Ophthalmology}

\title{
Coincidence of Inflamed Conjunctival Carcinoma in situ and Primary Pterygium
}

\author{
Hiroaki Endo ${ }^{a}$ Satoru Kase ${ }^{a, b} \quad$ Yasuo Suzuki $^{a} \quad$ Manabu Kase $^{a}$ \\ ${ }^{a}$ Department of Ophthalmology, Teine Keijinkai Hospital, Sapporo, Japan; ${ }^{b}$ Department of \\ Ophthalmology, Hokkaido University Graduate School of Medicine, Sapporo, Japan
}

\section{Keywords}

Pterygium · Carcinoma in situ · Interferon alfa-2b · Histopathology

\begin{abstract}
Background: We report a rare case of carcinoma in situ (CIS) in conjunction with a primary pterygium that exhibited characteristic angiographic and histopathological findings. Case: A 78-year-old man presented with a pterygium and a whitish tumor adjacent to the pterygium in his right eye. Indocyanine green angiography displayed that feeder vessels within the primary pterygium spread to the whitish tumor. The tumor and pterygial tissues were surgically removed. Histologically, the resected tissue contained CIS as well as squamous metaplasia. There was a marked inflammatory cell infiltration within the tumor and beneath the epithelium. Topical interferon alfa-2b was given 4 times per day for 2 months. The patient has been well without local recurrence of tumor or distal metastases to 54 follow-up months after surgery. Conclusions: Because CIS can occur adjacent to pterygial tissues, long-term followup is necessary in patients with pterygia.

\section{Introduction}

Conjunctival intraepithelial neoplasia contains dysplasia and carcinoma in situ (CIS) [1]. Ultraviolet rays and infections with viruses such as human papillomavirus and human im- 
Endo et al.: Coincidence of Inflamed Conjunctival Carcinoma in situ and Primary Pterygium

munodeficiency virus play an important role in the pathogenesis of conjunctival epithelial malignancies [2-4]. Pterygium is an invasion of the cornea by proliferation of abnormal (sub)conjunctival tissues together with a chronic inflammatory reaction. Conjunctival epithelial malignancy has a close relation to pterygium because of the fact that various proteins are expressed similarly in both epithelial cells [5] and CIS can coexist with pterygium [6]. However, there are no reports showing evidence that CIS is possibly arising from a primary pterygium itself or the adjacent tissue.

We herein report a rare case of CIS in conjunction with primary pterygium that exhibited characteristic angiographic and histopathological findings of inflammatory cell infiltration.

\section{Case Report}

A 78-year-old man presented with a conjunctival nodule in his right eye. He had a medical history of diabetes, hypertension, and cerebral hemorrhage. He has been under observation due to cataract and glaucoma by a nearby ophthalmologist from February 2012. In July 2012, slit lamp examination of the right eye revealed a primary nasal pterygium progressing to the cornea. Four months later, an elevated lesion was noted at the upper side of the pterygium. Since the elevated lesion became thicker with a rough surface and developed to the cornea, he was referred to our department on February 26, 2013. Visual acuity was $0.4(0.5$ $\times$ cyl $-3.00 \mathrm{DA} \times 30)$ OD and $0.3(0.4 \times \mathrm{cyl}-1.50 \mathrm{DA} \times 70)$ OS with normal intraocular pressure. Slit lamp examination demonstrated that the primary pterygial tissue was located in the nasal corneal limbus, where a whitish, bulging lesion with an irregular surface involved the upper side of the pterygium (fig. 1a). Anterior segment indocyanine green angiography (IA) displayed that the white mass was fed by neovessels derived from episcleral vessels (fig. $1 \mathrm{~b}$, arrowhead) as well as extended blood vessels originating from the pterygial tissue (fig. $1 \mathrm{~b}$, arrow). Systemic imaging modalities did not show any tumor lesions except in the right eye.

The whitish nodule was clinically diagnosed as CIS adjacent to a primary pterygium. Both lesions were resected with a safety margin (measuring $3 \mathrm{~mm}$ ), which was reconstructed using a conjunctival rotation flap on March 21, 2013. Histological findings of the tissue surgically removed were CIS as well as squamous metaplasia (fig. 2a, asterisks). In addition, there was an aggregation of plenty of lymphocytes forming lymphoid follicles beneath the epithelium (fig. 2a, arrows). At high magnification, neutrophil infiltration was markedly found to be present within the carcinomatous tissue (fig. $2 \mathrm{~b}$ ). The surgical margin of the corneal site was not free of tumor cells. Topical interferon (IFN) alfa-2b eyedrops were used as postoperative adjunct therapy 4 times a day for 2 months. On June 5, 2013, a biopsy was conducted from the corneal limbus where the tumor had been. Since the histology revealed no tumor cells, topical IFN eyedrop treatment was stopped. The patient was well without local recurrence of tumor or distal metastases up to December 2015.

\section{Discussion}

There are two possibilities of pathogenesis of CIS in this case: CIS may have arisen from the primary pterygium or it may have occurred de novo near the pterygium, subsequently inducing progression of the primary pterygium. In the latter case, there are some unique observations regarding the coincidence of CIS with pterygium: clinically, our patient had 
Case Reports in
Ophthalmology

Case Rep Ophthalmol 2016;7:208-212

DOI: $10.1159 / 000450824$

C 2016 The Author(s). Published by S. Karger AG, Basel www.karger.com/cop

Endo et al.: Coincidence of Inflamed Conjunctival Carcinoma in situ and Primary Pterygium

frequently been under observation by an ophthalmologist due to glaucoma, and the whitish lesion had initially been observed at the upper side of the primary pterygium, which developed CIS later. IA displayed that blood vessels originating from the primary pterygium spread to the whitish tumor. Moreover, histologically, intraepithelial carcinoma tissue was present together with squamous metaplasia consistent with pterygium. Moreover, this case showed marked inflammatory cell infiltration within the cancer tissue as well as within the subepithelial stroma. Therefore, we would like to diagnose the tumor as 'inflamed CIS' in this case. Although the CIS observed in this case has a close relationship to the primary pterygium based on histological and angiographic examination, it might be hard to determine whether the CIS really arose from the primary pterygium.

As treatment for CIS, complete resection is recommended conventionally [7, 8]. In recent years, local chemotherapy with IFN alfa- $2 \mathrm{~b}$ has been useful as an adjunctive therapy to surgical resection of CIS [7]. In our case, since histologically the corneal margin of the resected tumor was not free of tumor cells, topical IFN alfa-2b was added for 2 months following the surgery. Indeed, the histological examination proved no tumor cells in the biopsy specimens. Additional treatments were not required during the follow-up period of approximately 54 months after the surgery. In conclusion, we showed a rare case of inflamed CIS arising from a primary pterygium which was treated with surgical resection as well as postoperative topical IFN care. Because CIS can occur adjacent to pterygial tissues, long-term follow-up is necessary in patients with pterygia.

\section{Statement of Ethics}

The authors have no ethical conflicts to disclose.

\section{Disclosure Statement}

None of the authors has any conflict of interest related to the publication of this paper.

\section{References}

Lee GA, Hirst LW: Ocular surface squamous neoplasia. Surv Ophthalmol 1995;39:429-450.

Lee GA, Williams G, Hirst LW, et al: Risk factors in the development of ocular surface epithelial dysplasia. Ophthalmology 1994;101:360-364.

3 Scott IU, Karo CL, Nuovo GJ: Human papillomavirus 16 and 18 expression in conjunctival intraepithelial neoplasia. Ophthalmology 2002;109:542-547.

-4 Karp CL, Scott IU, Chang TS, et al: Conjunctival intraepithelial neoplasia. A possible marker for human immunodeficiency virus infection? Arch Ophthalmol 1996;114:257-261.

5 Detorakis ET, Zaravinos A, Spandidos DA: Growth factor expression in ophthalmic pterygia and normal conjunctiva. Int J Mol Med 2010;25:513-516.

-6 Oellers P, Karp CL, Sheth A, et al: Prevalence, treatment, and outcomes of coexistent ocular surface squamous neoplasia and pterygium. Ophthalmology 2013;120:443-654.

7 Schechter BA, Koreishi AF, Karp CL: Long-term follow up of conjunctival and corneal intraepithelial neoplasia treated with topical interferon alfa-2b. Ophthalmology 2008;115:1291-1296.

-8 Birkholz ES, Goins KM, Sutphin JE, et al: Treatment of ocular surface squamous cell intraepithelial neoplasia with and without mitomycin C. Cornea 2011;30:37-41. 
Endo et al.: Coincidence of Inflamed Conjunctival Carcinoma in situ and Primary Pterygium
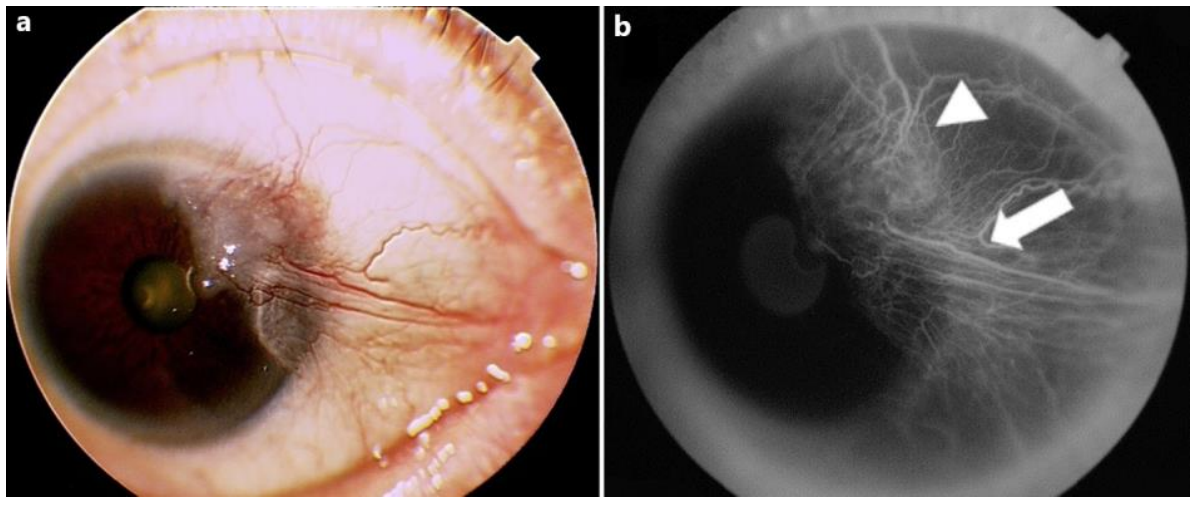

Fig. 1. Slit lamp examination (a) and anterior segment IA (b) in a case of conjunctival tumor. a The slit lamp image demonstrates that primary pterygial tissue is located in the nasal corneal limbus, where a whitish, elevated lesion with an irregular surface involves the upper side of the pterygium. b IA displays that the white mass was fed by neovessels derived from episcleral vessels (arrowhead) as well as by extended blood vessels originating from the pterygial tissue (arrow). 


\section{Case Reports in Ophthalmology}

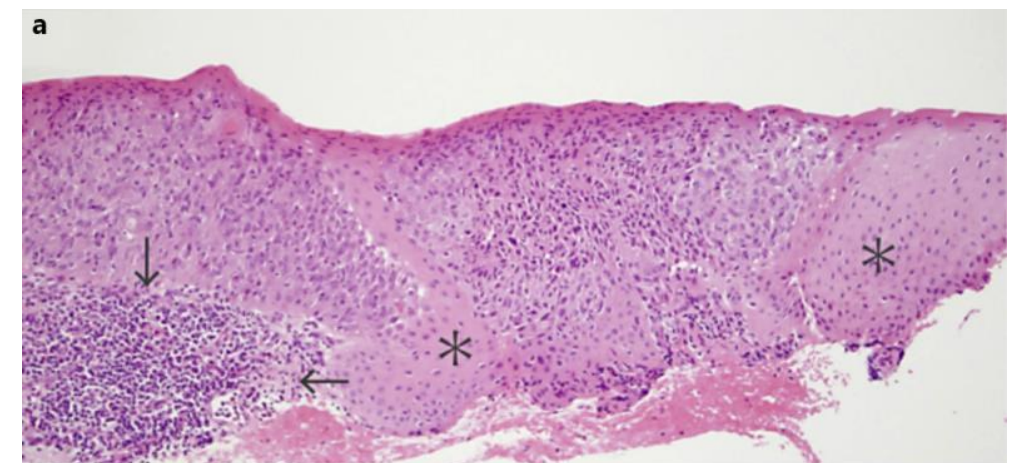

b

Endo et al.: Coincidence of Inflamed Conjunctival Carcinoma in situ and Primary Pterygium

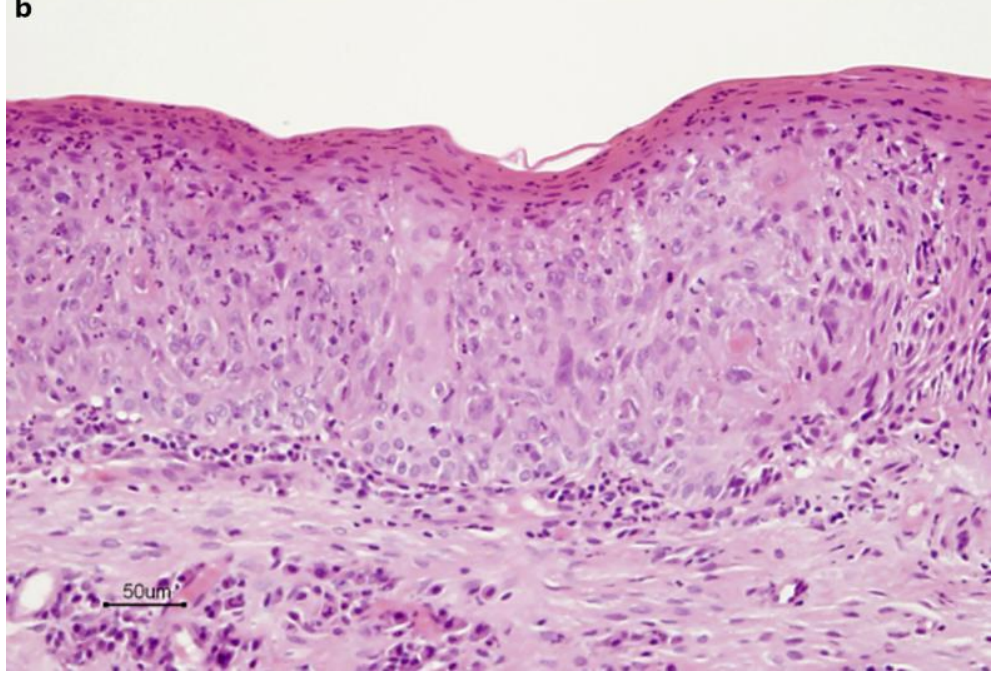

Fig. 2. Histopathological findings in the excised tissue of the conjunctival tumor. a Hematoxylin and eosin staining shows CIS as well as squamous metaplasia. There is an aggregation of plenty of lymphoid cells forming lymphoid follicles beneath the epithelium (arrows), where squamous metaplasia is involved (stars). b At high magnification, neutrophils are shown to have markedly infiltrated the carcinomatous tissue. Scale bar $=50 \mu \mathrm{m}$. 